\title{
Modeling and High-Resolution-Imaging Studies of Water-Content Profiles in a Polymer-Electrolyte-Fuel-Cell Membrane-Electrode Assembly
}

\author{
A. Z. Weber ${ }^{\mathrm{a}, *}$ and M. A. Hickner ${ }^{\mathrm{b}}$ \\ ${ }^{a}$ Environmental Energy Technologies Division, Lawrence Berkeley National Laboratory, Berkeley, CA 94720, USA. \\ ${ }^{\mathrm{b}}$ Department of Materials Science and Engineering, The Pennsylvania State University, University Park, PA 16802, USA.
}

Water-content profiles across the membrane electrode assembly of a polymer-electrolyte fuel cell were measured using high-resolution neutron imaging and compared to mathematicalmodeling predictions. It was found that the membrane held considerably more water than the other membrane-electrode constituents (catalyst layers, microporous layers, and macroporous gas-diffusion layers) at low temperatures, 40 and $60^{\circ} \mathrm{C}$. The water content in the membrane and the assembly decreased drastically at $80^{\circ} \mathrm{C}$ where vapor transport and a heat-pipe effect began to dominate the water removal from the membrane-electrode assembly. In the regimes where vapor transport was significant, the through-plane water-content profile skewed towards the cathode. Similar trends were observed as the relative humidity of the inlet gases was lowered. This combined experimental and modeling approach has been beneficial in rationalizing the results of each and given insight into future directions for new experimental work and refinements to currently available models.

Keywords: Polymer-electrolyte fuel cell; water transport; neutron imaging; mathematical model; thermal management *Corresponding author, phone: 510-486-6308, fax: 510-486-7303, email: azweber@lbl.gov 


\section{Introduction}

Gaining insight into the liquid-water distribution across the thickness of a polymerelectrolyte-fuel-cell (PEFC) membrane electrode assembly (MEA) has been the topic of many investigations in recent years. The experimental tools and mathematical models have become sufficiently advanced that detailed investigations of local-scale phenomena in the complicated environment of a fuel cell become reasonable. The advent of imaging techniques that are able to probe phenomena with micro-scale resolution [1-3] and the ability and understanding to simulate transport under operating conditions [4-10] has made for very fruitful research in the fuel-cell community.

In an earlier publication, high-resolution neutron imaging was used to investigate the through-plane liquid-water-content profile [11]. In that article, it is conjectured that the dominant mode of water transport through the various fuel-cell layers is due to evaporation and movement of water through the gas phase at high cell temperatures and/or high current densities. This heat-pipe effect has been shown through modeling to occur in fuel cells, and can be especially important in gas-diffusion layers (GDLs) or diffusion media (DM) $[12,13]$. In this paper, mathematical modeling is utilized to investigate this coupled-thermal-and-watermanagement issue in detail, and to explain some interesting qualitative features such as the location of the point of maximum liquid-water content as a function of operating conditions. In addition, imaging results and analysis for various temperatures and non-fully-humidified feeds are given. 


\subsection{Heat-pipe-effect simple analysis}

The heat-pipe effect occurs where evaporation of water from the cathode induces a watervapor flux from the cathode catalyst layer towards the cathode gas flow channel (GFC), which oxygen must move against to reach the cathode catalyst layer. This counter-current transport effect, combined with the reduction of oxygen partial pressure in the cathode feed stream due to the addition of water vapor, decreases the oxygen flux to the catalyst layer leading to a limiting current behavior.

To examine this effect in more detail, a simple back-of-the-envelope analysis can be done. Starting with the Stefan-Maxwell equations and assuming isobaric conditions, one can write expressions for the water-vapor flux and the oxygen mole-fraction gradient in a GDL with uniform properties (including saturation level) of [14, 15]

$$
\mathbf{N}_{\mathrm{w}}=-\frac{1}{1-y_{\mathrm{w}}}\left(\nabla y_{\mathrm{w}} \frac{p D_{\mathrm{w}, \text { air }}^{\mathrm{eff}}}{R T}+y_{\mathrm{w}} \frac{\mathbf{i}}{4 F}\right)
$$

and

$$
\nabla y_{\mathrm{O}_{2}}=\left(y_{\mathrm{O}_{2}} \mathbf{N}_{\mathrm{w}}+y_{\mathrm{w}} \frac{\mathbf{i}}{4 F}\right) \frac{R T}{p D_{\mathrm{w}, \mathrm{O}_{2}}^{\text {eff }}}+\left(1-y_{\mathrm{w}}-y_{\mathrm{O}_{2}}\right) \frac{\mathbf{i}}{4 F} \frac{R T}{p D_{\mathrm{O}_{2}, \mathrm{~N}_{2}}^{\text {eff }}}
$$

respectively, where $\mathbf{N}_{\mathrm{w}}$ is the water-vapor flux, $\mathbf{i}$ is the current density, $R$ is the ideal-gas

constant, $T$ is absolute temperature, $D_{\mathrm{w}, \mathrm{air}}^{\mathrm{eff}}$ is the effective binary diffusion coefficients and is corrected for gas-phase volume fraction and tortuosity, $p$ is the total gas-phase pressure, and $y_{i}$ is the mole fraction of species $i$. In the above expression for water, a pseudo binary approximation is used, and Faraday's law has been used to substitute the oxygen and nitrogen fluxes. For the heat-pipe effect, the mole-fraction gradient for water-vapor is given by the vapor-pressure 
gradient induced by the temperature gradient if local equilibrium is assumed. The actual temperature gradient will depend on the heat generation (potential and current density), thermal conductivities, water evaporation rates, etc. Using the above equations, the water-vapor flux and oxygen mole-fraction drop are calculated for different temperature gradients and cell temperatures, as shown in Figure 1.

From Figure 1(a), one can see that only a small temperature gradient is necessary to remove the electrochemically generated water, especially at $80^{\circ} \mathrm{C}$. Such an effect can be the main driver of water out of the cell which is beneficial in avoiding liquid-water flooding. However, in Figure 1(b) and (c), one can see how the temperature gradient reduces performance by lowering the oxygen concentration, especially at higher temperatures as the vapor pressure depends exponentially on temperature. Figure 1(b) shows that both the dilution effect at the higher temperature and the thermally induced water-vapor gradient decrease the oxygen mole fraction at the catalyst layer, as does higher current densities, in agreement with observed polarization performance. Figure 1(c) also demonstrates the strong percentage change in oxygen concentration that the heat-pipe effect can cause, where a value of 0 corresponds to no thermal gradient. Of course, the analysis leading to Figure 1 is simplified and ignores the liquid water flux, total gas-pressure changes, etc., but it is insightful and demonstrates the strong influence of thermal effects on water management and hence overall fuel-cell performance.

\section{Experimental and Theory}

The domain of both the experiments and the model is the same and detailed previously [11]. It consists of a high aspect ratio PEFC with an active area of $2.1 \times 7.7 \mathrm{~cm}$, of which the middle length of about $2 \mathrm{~cm}$ is imaged. The fuel cell is oriented with the gas inlets at the top of the cell 
and the single-channel serpentine flow path with $1 \mathrm{~mm}$ channel and land widths following gravity in a co-flow arrangement. Experiments were conducted under stoichiometric cathode gas flow control (flow rate adjusted relative to current production) at all current densities with the back pressure on the cell maintained at $10 \mathrm{kPa}$ on both anode and cathode regardless of flow rate. The anode flow rate was maintained at $480 \mathrm{std} . \mathrm{cm}^{3} \mathrm{~min}^{-1}$ for all tests and both anode and cathode inlet gas streams were humidified by dew point control. Membrane electrode assemblies were in-house fabricated catalyst coated Nafion $112^{1}$ membranes (Dupont, Wilmington, DE) with catalyst loadings of $1 \mathrm{mg} \mathrm{cm}^{-2}$ platinum on both anode and cathode. The catalyst used was E-TEK (BASF Future Business GmbH, Ludwigshafen) $40 \%$ by weight platinum on XC-72 carbon black. The GDLs used were carbon cloth with integral micro-porous layer, E-TEK LT1400W. All quantitative measurements and images were processed using a dry reference image and background subtraction. Experimental water contents are reported on a volume fraction basis in milliliters of water per cubic centimeter of volume imaged. Each pixel in the images corresponds to dimensions of 16.4 x $16.4 \mu \mathrm{m}$ (as measured using a fiducial maker in the image analysis). The total volume imaged per pixel is $5.6 \times 10^{-6} \mathrm{~cm}^{3}$ given a cell width of $2.1 \mathrm{~cm}$ parallel to the beam direction. The one root-mean-square deviation of the liquid water thickness was $0.003 \mathrm{~mm}$ for an 811 pixel region, which equates to an uncertainty of $1.2 \%$ in the experimental volume fractions reported here.

The modeling was achieved with pseudo 2-D (1+1-D) simulations using the code developed by Weber and Newman [13]. The domain consists of the layers and thicknesses as described above, and the operating conditions were the same as the experimental ones. The presented

\footnotetext{
${ }^{1}$ Certain trade names and company products are mentioned in the text or identified in an illustration in order to adequately specify the experimental procedure and equipment used. In no case does such identification imply recommendation or endorsement by NIST, nor does it imply that the products are necessarily the best available for this purpose.
} 
modeling distributions represent calculations at the middle point of the cell to match the imaging location. Because most of the properties of the cell materials have not been measured, average values from the literature are taken and the active surface area and effective permeability of the GDL are fit to the polarization-curve data of Hickner et al. [11] Overall, the modeling results are used to explain the observed trends and results qualitatively, since there are too many unknown materials parameters (e.g. pore size distribution, fraction of hydrophilic and hydrophobic pores, etc.) to make strong quantitative comparisons.

\section{Results and Discussion}

Experimental water-content values from neutron imaging are shown in Figure 2. A comparison among the temperatures shows that the position of the maximum water content and the water-content profile across the GDL changes significantly as the temperature of the cell is raised towards $80^{\circ} \mathrm{C}$. Furthermore, there is a corresponding decrease in the GDL water contents as the current density is increased. This is especially apparent in the $80^{\circ} \mathrm{C}$ case. To understand this effect and set the stage for further discussions, the simulated temperature profile is shown in Figure 3 for the $60^{\circ} \mathrm{C}$ case.

From the figure, it is clear that at high current densities, as discussed later, the temperature gradients are significant enough to alter the water distributions and cause evaporation of the liquid water. As discussed in Figure 1, this will have a greater impact as the cell temperature is increased. As expected, the peak temperature occurs within the cathode catalyst layer and the gradients are primarily caused by the relatively thick GDLs [16]. One also sees that the temperature at the GFC also increases at high current densities, thereby decreasing the humidity of the incoming gases. This effect also drives water movement toward the anode, explaining 
why the water content levels in the anode GDL rise as the current density is increased for the 60 and $40^{\circ} \mathrm{C}$ cases.

At $80{ }^{\circ} \mathrm{C}$, the water-content profile in Figure 2 has a distinct shape that lacks the increases near the GFC / GDL boundaries, indicating that the water removal mechanism is probably different (e.g., evaporation instead of droplet emergence). To understand these profiles better, simulations are carried out. The simulation and experimental comparisons are done at $0.75 \mathrm{~A}$ $\mathrm{cm}^{-2}$ since that case corresponds to similar cell potentials for all three temperatures $(0.57,0.62$, and $0.63 \mathrm{~V}$ for 40,60 and $80^{\circ} \mathrm{C}$, respectively), meaning that the heat generation is also similar [11].

There are two ways that the simulation results are presented, as capillary pressures or as saturations (water volume fractions). From an understanding and physics standpoint, the capillary pressure, as defined by the liquid pressure minus the gas pressure, is preferred. The reason is that the capillary pressure is more physics-dependent than the saturation as it depends directly on the liquid and gas pressures and not also on the material and fluid properties. As detailed in the Appendix, the saturation is derived from the capillary pressure (i.e., it is a dependent property) [17]. However, both quantities are linked and solved for in the problem. While the saturation does allow a direct comparison to the experimental data, such a translation requires assuming some perhaps unknown parameters that change the absolute values of the water content, as discussed in the Appendix.

In terms of comparisons between the experimental data and simulation values, one does not expect strong quantitative agreement; qualitative trends are the goal at present. The reasons for the discrepancies between the data and simulation are discussed in more detail below, but are mainly due to the relative simplicity of the pseudo 2-D model being used, including the 
quantification of the saturation, and experimental error in resolving water content at high resolution. The former means that the effect of the lands are neglected, and thus results for the model should be higher than expected. Furthermore, the low saturations implied by the data in Figure 2 mean that using traditional two-phase-flow treatments may be inappropriate because the water-transport mechanism may be more that of drops and movement along fibers rather than in (cylindrical) pores. The experimental error is related to the fact that resolving water content at high spatial resolution is complicated by a significant amount of scattering as compared to the spatial dimensions of the pixels. This scattering decreases the experimentally observed liquid water content. Regardless, the comparison between model and data does allow one to get a sense of the modeling areas for improvement and explanation of some of the data trends such as the interplay between thermal and water management.

The capillary-pressure and the liquid-volume profiles are given in Figure 4 and Figure 5, respectively, for the three temperatures at $0.75 \mathrm{~A} \mathrm{~cm}^{-2}$ and fully humidified feeds. In Figure 4, lower capillary pressures denote lower saturations and less liquid water in general. There is a break in the values for the membrane since the water content is defined differently (see the Appendix). These discontinuities do not occur in the experimental data because the true interfaces are not as sharp and thin as those in the simulation and are being averaged over many locations along the interface.

From the predicted profiles at 40 and $60^{\circ} \mathrm{C}$, the high concentration of liquid water in the region between the microporous layers can be explained by the fact that these layers inhibit liquid water transport, thus concentrating it in the membrane and catalyst layers. For the GDL water profiles, the MPL acts as a barrier for liquid-water transport. Liquid water is produced at the catalyst layer and transported through the MPL as a saturated vapor. This results in the steep 
drop in liquid content at the MPL / GDL interface as shown in Figure 4 and Figure 5. The saturated vapor moves through the GDL and begins to condense as the local temperature drops (i.e., the heat-pipe effect) leading to a recirculating flow where the liquid water then moves back toward the membrane. In the simulations, the dry region occurs when the liquid water no longer has enough pressure to move further into the warmer layer and it vaporizes. While the minimum in water content in the simulations occurs at the GDL / MPL boundary, it occurs further into the GDL in the experiments. This difference in the position of minimum water content between the model and experiment can be explained by the fact that the simulation assumes uniform independent properties (i.e., porosity, pore-size and wettability distributions, etc.) within the GDL. This assumption is undoubtedly an idealization, where due to compression and inherent nonuniformities, the real situation has variable properties. In fact, some preliminary X-ray tomographic data from us (not shown) hint that the GDL may exhibit inherent surface densification due to manufacturing. Having variable properties would alter the location of the minimum water-content value [18], and should be considered in future PEFC modeling.

The complicated GDL water-content profiles also are not fully captured using the simulation because a pseudo 2-D approach is utilized, wherein the ribs are neglected. While this greatly simplifies the computational cost and increases model robustness, it also does not allow for certain extrema to be captured. For example, water condenses preferentially on the ribs due to their lower temperature, which is probably the main cause of the maxima in the GDLs near the GFCs, along with imprecise interface identification in the experiment. The neglect of the rib/channel effects coupled with the use of uniform structural properties mean that the gradients in the simulations are inherently monotonic and unable to predict the minimum in the cathode GDL. 
In comparing the 40 and $60^{\circ} \mathrm{C}$ cases, both the predicted and simulated values shows the same trends. At $40^{\circ} \mathrm{C}$, the cathode and anode GDLs have higher liquid-water contents in agreement with the analysis presented in Figure 1 due to a decrease in the heat-pipe effect. The lower temperature and less importance of the temperature gradient causes the MPLs to both contain more liquid and for a different flow profile to develop as seen in the capillary-pressure curves in Figure 4. Due to higher liquid-water amount in the MPLs, the anode GDL minimum is not as prominent and a shoulder exists in the cathode MPL due to the similar values between the MPL and the CL. In terms of trend deviation, besides the lack of extrema as mentioned above, the greatest discrepancy is that of the anode MPL at $40^{\circ} \mathrm{C}$. This can be explained by the fact that the model is either not predicting as much water back-transport or is not as resistive towards water uptake in the anode side of the membrane as in the experiment. The former could be caused by a lack of considering membrane compression decreasing the membrane thickness.

The water-content profiles at $80^{\circ} \mathrm{C}$ exhibit a different shape than those at 40 and $60^{\circ} \mathrm{C}$. The simulations clearly demonstrate a reduced water content that is due to evaporative removal beginning to dominate the total water removal from the cell at higher temperatures. The maximum water amount unexpectedly shifts position at $80^{\circ} \mathrm{C}$ in both the data and the simulations. The reason for this shift is because the water-vapor in the cathode MPL condenses at the MPL / GDL interface due to the much larger and more hydrophilic pores in the GDL. This then causes liquid-water flow both out of the cell and back into the MPL as can be seen in the capillary pressure profiles in Figure 4. The maximum may occur nearer the cathode catalyst layer than predicted, although this again will depend on such properties as porosity, membrane volume fraction, catalyst-layer thickness, etc., which are probably not uniform. For the anode GDL, the model predicts that there is essentially no liquid water, whereas the data shows some 
small amounts. The reason is that the analysis assumes cylindrical pores that are either empty or filled, whereas in reality there is probably water on the fibers (especially any hydrophilic regions) and droplets within the medium, which the model does not consider explicitly.

A quantitative comparison between Figure 2 and Figure 5 shows that while the values are within reason for most of the components, there is a large discrepancy between the membrane values at 40 and $60^{\circ} \mathrm{C}$. The deviations in the catalyst layers are also directly related to the membrane water content in those layers. The quantitative discrepancy is probably due to both an inaccurate membrane model and some experimental error. The membrane model used, as proposed by Weber and Newman [19], is probably insufficient in terms of dealing with the vapor and liquid interfaces. For example, it uses values for interfacial mass-transfer coefficients that are large and thus do not represent a significant resistance for transport to/from the vapor phase, counter to some recent experimental studies [20,21]. Making this value smaller would result in larger water hold-up in the anode and perhaps a drier membrane, which could explain why the profile at the anode MPL is wrong at $40^{\circ} \mathrm{C}$.

Furthermore, the model assumes that a sufficient value of the chemical potential of water can result in a fully liquid-equilibrated membrane, even if the membrane interface is exposed to saturated vapor. This does not necessarily make physical sense, and one can change the model to use a so-called phase-in-contact approach. This approach effectively sets a maximum on the water content on the membrane depending on how much of the interface is exposed to liquid water versus water vapor. Applying this approach to the simulation results in Figure 5 reduces the amount of water in the membrane roughly by half, as show in Figure 6 for 60 and $80^{\circ} \mathrm{C}$. Figure 6 also smoothes the interfaces to make a more continuous water profile. The smoothing is done by simple averaging assuming a 5 to $10 \mu \mathrm{m}$ interfacial zone (which is still less than the 
pixel resolution). Finally, the above changes to the membrane model would not change the results at $80^{\circ} \mathrm{C}$ substantially, which demonstrate fairly good quantitative agreement.

In terms of experimental issues, as noted above, there is a significant issue of deconvoluting the scattering seen under high spatial resolutions and with membrane water uptake. Work is currently underway at NIST to quantify this error. However, the shape of the water-content curves will remain unchanged although the relative differences between the maximum and minimum values may change. Figure 6 clearly demonstrates that the model and experiment are in adequate agreement in terms of many of the qualitative trends such as the existence of extrema and the movement of the water-content maximum. For better quantitative agreement, in addition to verification of the experimental results, model improvements should be made consisting of a more refined and validated membrane-interface model, a full 2-D model including rib/channel effects, and a droplet or similar low-water-content two-phase-flow submodel for the GDLs. Furthermore, one should also account for any possible membrane compression.

In the rest of the paper, the genesis of the above theoretical water profiles is further investigated with a focus on the interplay between water and thermal management. In addition, examination of data and some modeling insights for non fully-humidified inlets is also discussed. To explore the heat-pipe effect in more detail, Figure 7 displays the liquid- and vapor-water fluxes in the anode and cathode diffusion media (GDLs and MPLs). For the anode side, all of the liquid fluxes are positive and small indicating that the majority of the water moves through the vapor phase. The vapor fluxes are negative indicating that the heat-pipe effect causes flow reversal at all temperatures, although this effect becomes less significant as the temperature is decreased. The lowering of this effect causes more liquid water buildup in the layers at the lower temperatures. 
The above impact of temperature and the heat-pipe effect is more evident when one examines the cathode-diffusion-medium flux profiles in Figure 7. For all temperatures, almost all of the water-vapor fluxes are positive indicating that flow reversal occurs for the vapor phase (one expects that the flow should be negative, i.e., towards the membrane). Comparing the profiles demonstrates that while at $40^{\circ} \mathrm{C}$, over $80 \%$ of the water is moving in the liquid phase in the GDL, this amount decreases to around $60 \%$ at $80^{\circ} \mathrm{C}$. Even more interesting is within the cathode MPLs, where again the liquid-water flux is much greater at $40^{\circ} \mathrm{C}$ than at the other temperatures. This explains the broader peak of the water content seen in Figure 2 and Figure 5. Also, the interesting shape of the water content in those figures for the $80^{\circ} \mathrm{C}$ is shown in that there is a negative flux of water in the MPL, indicating that at the MPL / GDL interface there is a maximum in liquid water since it is moving away in both directions. Finally, for all the temperatures, as one approaches the hotter catalyst layer, more of the water is being carried in the vapor phase.

As a final underscore to the relation between water and thermal management, Figure 8 gives simulated capillary-pressure profiles where the GDL (both macroporous and microporous) thermal conductivity changes at $60^{\circ} \mathrm{C}$ and $0.75 \mathrm{~A} \mathrm{~cm}^{-2}$. The figure shows that doubling the thermal conductivity results in less vaporization in the MPL and slightly higher water contents throughout the cell. Conversely, halving the thermal conductivity results counter-intuitively in larger water contents in the anode side and smaller ones in the cathode side and membrane. The larger water contents in the anode are due to a greater heat-pipe effect and recirculation of water due to the larger temperature gradients. Such an effect decreases the water on the cathode since more can be removed by evaporation. Overall, the cell design, operating conditions, and 
materials can have larger impacts on both the quantitative and qualitative water-content profiles due to the interplay between thermal and water management.

For all of the above analysis, the feed gases are fully humidified. This creates a situation wherein the heat-pipe effect and associated thermal management can be dominant since the gases are saturated and thus the vapor-pressure profile established by the temperature gradient impacts performance. To examine the generality of the above trends, it is worthwhile to investigate what happens with different relative-humidity gas feeds. Figure 9 shows the liquid-water-content profiles as a function of relative humidity of the anode and cathode inlet gas streams for the cell at $60^{\circ} \mathrm{C}$ and $1 \mathrm{~A} \mathrm{~cm}^{-2}$. A similar trend to that of increasing cell temperature (Figure $2 \mathrm{~b}$ ) is seen with decreasing relative humidity. Namely, as the water content decreases, the distribution across the layers skews towards the cathode. This reinforces the concept of the membrane dominating the water content when significant hydration is maintained (at low temperature or high relative humidity), whereas under sub-saturated conditions the liquid water is found more on the cathode side of the cell. Additionally, the water saturations measured by high-resolution neutron radiography are on the lower end of what is anticipated from the models.

For the lower relative-humidity cases, simulations are carried out (not shown) for similar conditions and the following conclusions made. Neither the anode nor cathode GFCs become fully humidified, meaning that the heat-pipe effect is not dominant. The anode subsaturation causes more membrane dehydration and a flatter and lower water-content profile since more water is moving in the vapor-equilibrated state rather than the liquid-equilibrated one (the membrane water content changes less in the vapor-equilibrated mode for a given flux). The subsaturation also results in a downward gradient in the water content and capillary pressure, similar to the case at $80^{\circ} \mathrm{C}$ (see Figure 4). The cathode level of subsaturation is less than the 
anode one since the stoichiometry is much lower and water is produced at the cathode. However, since it is not fully humidified, the water movement is mainly in the vapor phase, with small amounts in the hydrophilic domains of the GDL, resulting in the profile shape seen in the figure.

\section{Conclusions}

Experimental and modeling comparisons were conducted for liquid-water-content profiles across the fuel-cell sandwich composed of gas-diffusion layers, microporous layers, catalyst layers, and Nafion 112 membrane. The experiments utilized high-resolution neutron imaging and conditions of variable feed humidity, cell temperature, and current density. A pseudo 2-D model was used to explain many of the observed features and trends seen with the neutron radiography. It was determined that there is a strong heat-pipe effect where the water is moving primarily through the vapor phase and condenses along the temperature gradient. This type of behavior causes liquid-water profiles with maxima and minima in the diffusion media, especially with more humidified feeds. At $80^{\circ} \mathrm{C}$, there is a shift in the maximum water content towards the cathode side due to capillary condensation and some drying out of the gases. The explanations given represent a complex water-transport pathway composed of multiphase flow and coupled thermal and water management. Furthermore, the low saturations observed may mean that traditional continuum flow is not occurring, and droplet or a type of percolation flow is through single pores which connect the two higher-concentration areas of liquid water. For a more quantitative analysis, model improvements and experimental verification of the data are required.

The suggested model improvements include consideration of the droplet or low-water-content two-phase-flow, a more rigorous and validated approach towards treating the membrane 
interface in contact with liquid water and water vapor, and altering the domain to include the rib and channel.

\section{Acknowledgements}

This work was supported by the NIST Ionizing Radiation Division, the Director's office of NIST, the NIST Center for Neutron Research, The Pennsylvania State University Materials Research Institute, the Penn State Institutes of Energy \& the Environment, and support provided to Lawrence Berkeley National Laboratory by Toyota Motor Engineering and Manufacturing, North America and Plug Power, Inc., under contracts LB08003874 and LB07003357, respectively. This work was supported by the U.S. Department of Energy under Contract Number DE-AC02-05CH11231. 


\section{Appendix}

To calculate the water-volume amount from the capillary pressure, one starts with determining critical radii to be used as integration limits

$$
r_{c, h}=-\frac{2 \gamma \cos \theta_{h}}{p_{\mathrm{L}}-p_{\mathrm{G}}}=-\frac{2 \gamma \cos \theta_{h}}{p_{\mathrm{C}}}
$$

where $\gamma$ is the surface tension of water, is the $\theta_{h}$ is the contact angle between water in air and solid of type $h$, which is either hydrophilic (HI) or hydrophobic (HO). For $p_{C} \leq 0, \infty \leq r_{c, \mathrm{HI}}>0$ and $r_{c, \mathrm{HO}}=\infty$, and for $p_{C} \geq 0, r_{c, \mathrm{HI}}=\infty$ and $\infty \leq r_{c, \mathrm{HO}}>0$. If one assumes a binary hydrophobicity distribution (one hydrophilic and one hydrophobic), then the liquidvolume can be obtained by

$$
S_{\mathrm{LV}}=\varepsilon \frac{\int_{0}^{r_{c, \mathrm{HI}}} V_{\mathrm{HI}}(r) \mathrm{d} r+\int_{r_{c, \mathrm{HO}}}^{\infty} V_{\mathrm{HO}}(r) \mathrm{d} r}{\int_{0}^{\infty} V_{\mathrm{HI}}(r) \mathrm{d} r+\int_{0}^{\infty} V_{\mathrm{HO}}(r) \mathrm{d} r}+S_{\mathrm{irr}}
$$

where $\varepsilon$ is the porosity of the medium, $V_{h}$ is the pore volume of type $h$, and $r$ is the pore radius, and $S_{\text {irr }}$ is the irreducible saturation, a constant that accounts for irregular cavities (e.g., inkbottle well) that are not described by the distribution. If the same distribution is used for both the hydrophilic and hydrophobic pores, then the above equation can be rewritten as

$$
S_{\mathrm{LV}}=\varepsilon\left[f_{\mathrm{HI}} \int_{0}^{r_{c, \mathrm{HI}}} V(r) \mathrm{d} r+\left(1-f_{\mathrm{HI}}\right) \int_{r_{c, \mathrm{HO}}}^{\infty} V(r) \mathrm{d} r\right]+S_{\mathrm{irr}}
$$

where $f_{\mathrm{HI}}$ is the fraction of hydrophilic pores, which is then also the liquid pore saturation at a zero capillary pressure. 
To determine the membrane water-volume amount, one needs the water content or moles of water per mole of sulfonic-acid site, $\lambda$, of the membrane. Because local equilibrium is assumed, the capillary pressure in the membrane can be used in the following manner. First, if the capillary pressure is positive, then the fraction of expanded channels can be calculated, and $\lambda$ is given by

$$
\lambda=\left.\lambda_{\mathrm{v}}\right|_{a_{\mathrm{w}}=1}+\left(22-\left.\lambda_{\mathrm{v}}\right|_{a_{\mathrm{w}}=1}\right) f_{\text {expanded }}
$$

where $\left.\lambda_{\mathrm{v}}\right|_{a_{\mathrm{w}}=1}$ is the $\lambda$ value for the membrane in equilibrium with saturated vapor at the given temperature. If the capillary pressure is less than zero, this can be changed into a water activity of

$$
a_{\mathrm{w}}=\exp \left(\frac{\overline{V_{\mathrm{w}}} p_{C}}{R T}\right)
$$

where $\overline{V_{\mathrm{w}}}$ is the partial molar volume of water. In the above expression, a secondary reference state of water at unit activity is applied. The activity can then be used to calculate $\lambda$ from a submodel or by an isotherm. Once $\lambda$ is known, it can be used to get the water-volume amount by

$$
S_{\mathrm{LV}}=\frac{\lambda \bar{V}_{\mathrm{w}}}{\bar{V}_{\mathrm{m}}+\lambda \bar{V}_{\mathrm{w}}}
$$

where $\overline{V_{\mathrm{m}}}$ is the partial molar volume of the dry membrane and free-swelling conditions are assumed.

Finally, the water content in the catalyst layer is the weighted sum of the water content in the membrane in the catalyst layer and that in the pores of the catalyst layer. 


\section{References}

[1] P.K. Sinha, P. Halleck, C.Y. Wang, Electrochemical and Solid State Letters, 9 (2006) A344.

[2] D.S. Hussey, D.L. Jacobson, M. Arif, J.P. Owejan, J.J. Gagliardo, T.A. Trabold, J. Power Sources, 172 (2007) 225.

[3] F. Xu, O. Diat, G. Gebel, A. Morin, J. Electrochem. Soc., 154 (2007) B1389.

[4] V.P. Schulz, J. Becker, A. Wiegmann, P.P. Mukherjee, C.Y. Wang, J. Electrochem. Soc., 154 (2007) B419.

[5] A.Z. Weber, J. Newman, Chemical Reviews, 104 (2004) 4679.

[6] C.Y. Wang, Chemical Reviews, 104 (2004) 4727.

[7] S. Litster, J.G. Pharoah, G. McLean, N. Djilali, J. Power Sources, 156 (2006) 334.

[8] J.J. Kowal, A. Turhan, K. Heller, J. Brenizer, M.M. Mench, J. Electrochem. Soc., 153 (2006) A1971.

[9] E.C. Kumbur, K.V. Sharp, M.M. Mench, J. Power Sources, 168 (2007) 356.

[10] M. Secanell, B. Carnes, A. Suleman, N. Djilali, Electrochim. Acta, 52 (2007) 2668.

[11] M.A. Hickner, N.P. Siegel, K.S. Chen, D.S. Hussey, D.L. Jacobson, M. Arif, J. Electrochem. Soc., 155 (2008).

[12] Y. Wang, C.Y. Wang, J. Electrochem. Soc., 153 (2006) A1193.

[13] A.Z. Weber, J. Newman, J. Electrochem. Soc., 153 (2006) A2205.

[14] R.B. Bird, W.E. Stewart, E.N. Lightfoot, Transport Phenomena, John Wiley \& Sons, Inc., New York, 2002.

[15] J. Newman, K.E. Thomas-Alyea, Electrochemical Systems, John Wiley \& Sons, New York, 2004. 
[16] M. Khandelwal, M.M. Mench, J. Power Sources, 161 (2006) 1106.

[17] A.Z. Weber, R.M. Darling, J. Newman, J. Electrochem. Soc., 151 (2004) A1715.

[18] J.H. Nam, M. Kaviany, Int. J. Heat Mass Transfer, 46 (2003) 4595.

[19] A.Z. Weber, J. Newman, J. Electrochem. Soc., 151 (2004) A311.

[20] P.W. Majsztrik, M.B. Satterfield, A.B. Bocarsly, J.B. Benziger, J. Membr. Sci., 301 (2007) 93.

[21] M.B. Satterfield, J.B. Benziger, J. Phys. Chem. B, 112 (2008) 3693. 


\section{Figure Captions}

Figure 1. Heat-pipe and reverse-water-vapor-gradient effects as a function of GDL temperature difference for a $250 \mu \mathrm{m}$ GDL with a 0.6 effective gas-phase volume fraction for cell temperatures of 40,60 , and $80^{\circ} \mathrm{C}$. (a) Water-vapor flux at $1 \mathrm{~A} \mathrm{~cm}^{-2}$ per water amount generated. (b) Oxygen mole fraction at the catalyst layer for two current densities. (c) Oxygen-mole-fraction decrease relative to the isothermal case.

Figure 2. Through-plane water-volume fractions for $40(+), 60(\mathrm{o})$, and $80(\mathrm{x}){ }^{\circ} \mathrm{C}$ cell temperatures at (a) 0.75 and (b) $1 \mathrm{~A} \mathrm{~cm}^{-2}$ and $100 \%$ RH gas feeds. The dashed lines indicate the GDL/MPL boundaries and the solid line is the geometric centerline.

Figure 3. Simulated temperature profiles as a function of current density from the anode to cathode GDL / GFC interfaces for a cell temperature of $60^{\circ} \mathrm{C}$ with $100 \% \mathrm{RH}$ feeds.

Figure 4. Simulated capillary-pressure profiles from the anode to the cathode GDL / gaschannel interfaces for $0.75 \mathrm{~A} \mathrm{~cm}^{-2}$ at 40,60 , and $80^{\circ} \mathrm{C}$ with $100 \% \mathrm{RH}$ feed gases. The break is due to different meaning of capillary pressure in the membrane. The lower the capillary pressure, the lower the water content, with larger negative values (outside of the membrane) being associated with water at its residual saturation (i.e., movement is by water-vapor alone).

Figure 5. Liquid-volume profiles for the cases in Figure 4. For the conversion, symmetric layers are assumed (e.g., same GDL properties on the anode and cathode), with the 
same hydrophilic and hydrophobic pore-size distributions, $\varepsilon_{\mathrm{GDL}}=0.6, \varepsilon_{\mathrm{MPL}}=\varepsilon_{\mathrm{CL}}=$ $0.3, \varepsilon_{\mathrm{Mem}}=0.4$ in the catalyst layers, $f_{\mathrm{HI}, \mathrm{GDL}}=0.1$, and $f_{\mathrm{HI}, \mathrm{CL}}=f_{\mathrm{HI}, \mathrm{MPL}}=0.06$.

Figure 6. Simulated (see Figure 5) liquid-volume profiles for the 60 and $80^{\circ} \mathrm{C}$ cases assuming a phase-in-contact membrane model and smoothing of the interfaces. The curves are overlaid on the experimental data from Figure 2.

Figure 7. Liquid- (black) and vapor- (grey) water-flux profiles for the anode and cathode diffusion medium (GDLs and MPLs) as a function of cell temperature for 100\% RH feed gases. The fluxes are made dimensionless by dividing by the water production rate at $0.75 \mathrm{~A} \mathrm{~cm}^{-2}$.

Figure 8. Simulated capillary-pressure profiles at $0.75 \mathrm{~A} \mathrm{~cm}^{-2}$ and $60^{\circ} \mathrm{C}$ for base, double, and half values of the GDL and MPL effective thermal conductivities with $100 \% \mathrm{RH}$ feeds.

Figure 9. Through-plane water-volume fractions for gas feeds of 100, 90, and 70\% RH at 60 ${ }^{\circ} \mathrm{C}$ for the anode and cathode respectively at $1 \mathrm{~A} \mathrm{~cm}^{-2}$. 


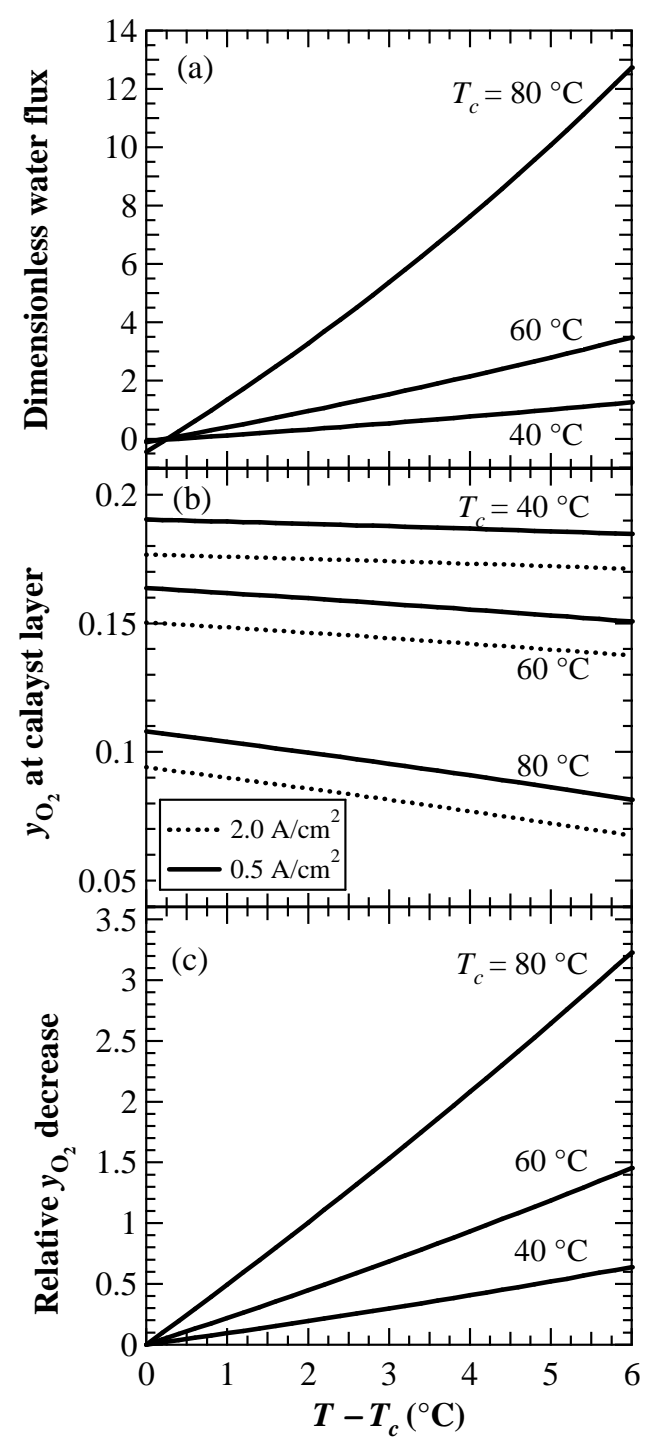

Figure 1. Heat-pipe and reverse-water-vapor-gradient effects as a function of GDL temperature difference for a $250 \mu \mathrm{m}$ GDL with a 0.6 effective gas-phase volume fraction for cell temperatures of 40,60 , and $80^{\circ} \mathrm{C}$. (a) Water-vapor flux at $1 \mathrm{~A} \mathrm{~cm}^{-2}$ per water amount generated. (b) Oxygen mole fraction at the catalyst layer for two current densities. (c) Oxygen-molefraction decrease relative to the isothermal case. 


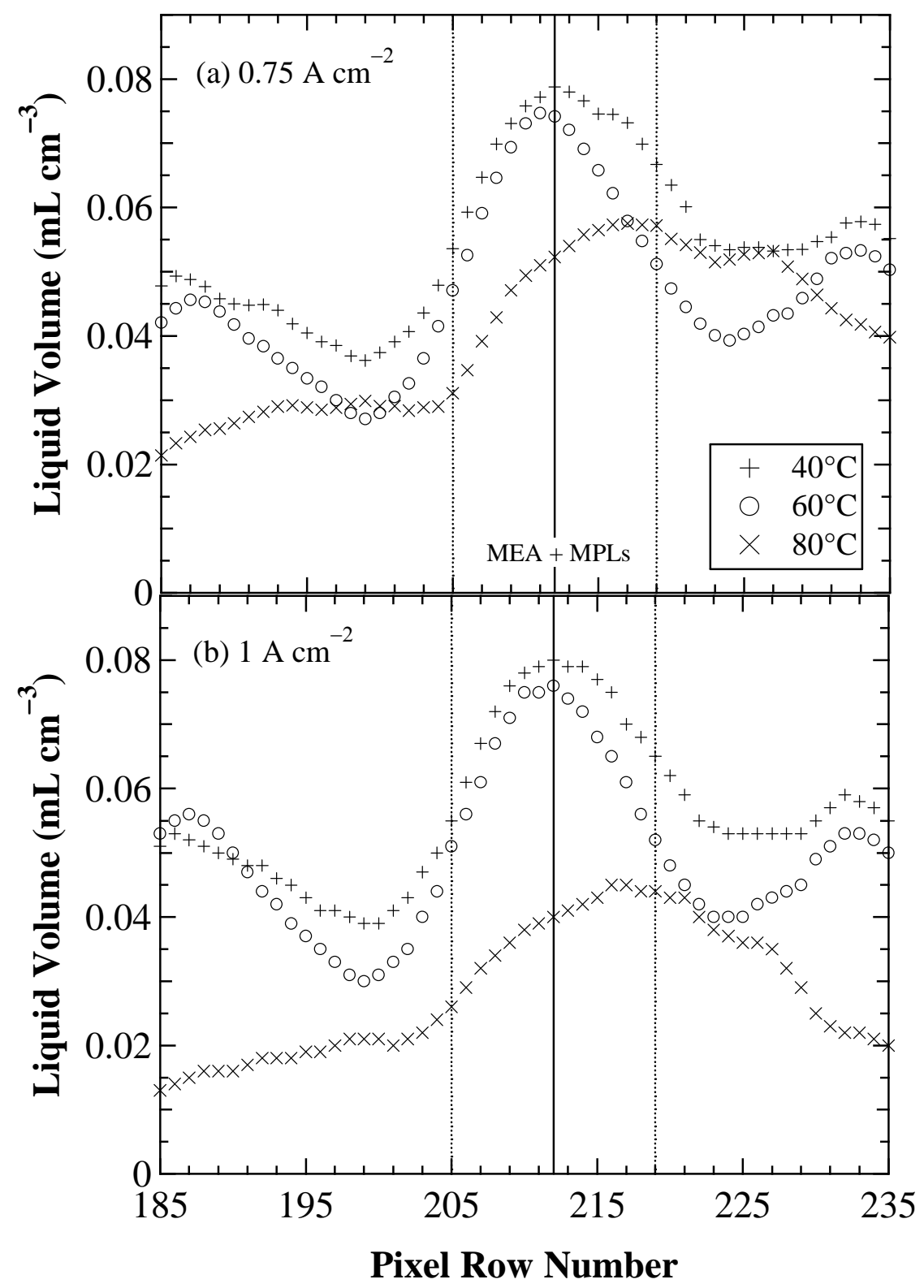

Figure 2. Through-plane water-volume fractions for $40(+), 60(\mathrm{o})$, and $80(\mathrm{x}){ }^{\circ} \mathrm{C}$ cell temperatures at (a) 0.75 and (b) $1 \mathrm{~A} \mathrm{~cm}^{-2}$ and $100 \% \mathrm{RH}$ gas feeds. The dashed lines indicate the GDL/MPL boundaries and the solid line is the geometric centerline 


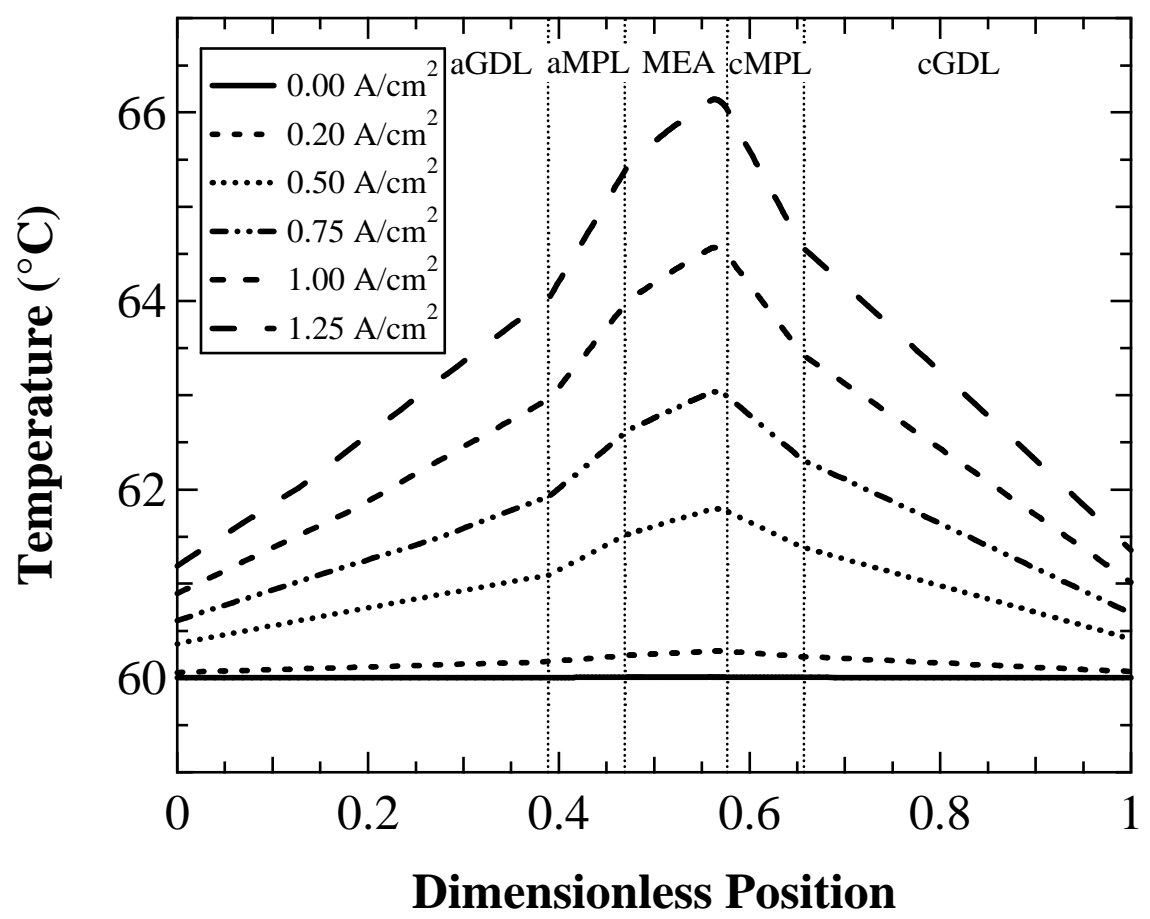

Figure 3. Simulated temperature profiles as a function of current density from the anode to cathode GDL / GFC interfaces for a cell temperature of $60^{\circ} \mathrm{C}$ with $100 \% \mathrm{RH}$ feeds. 


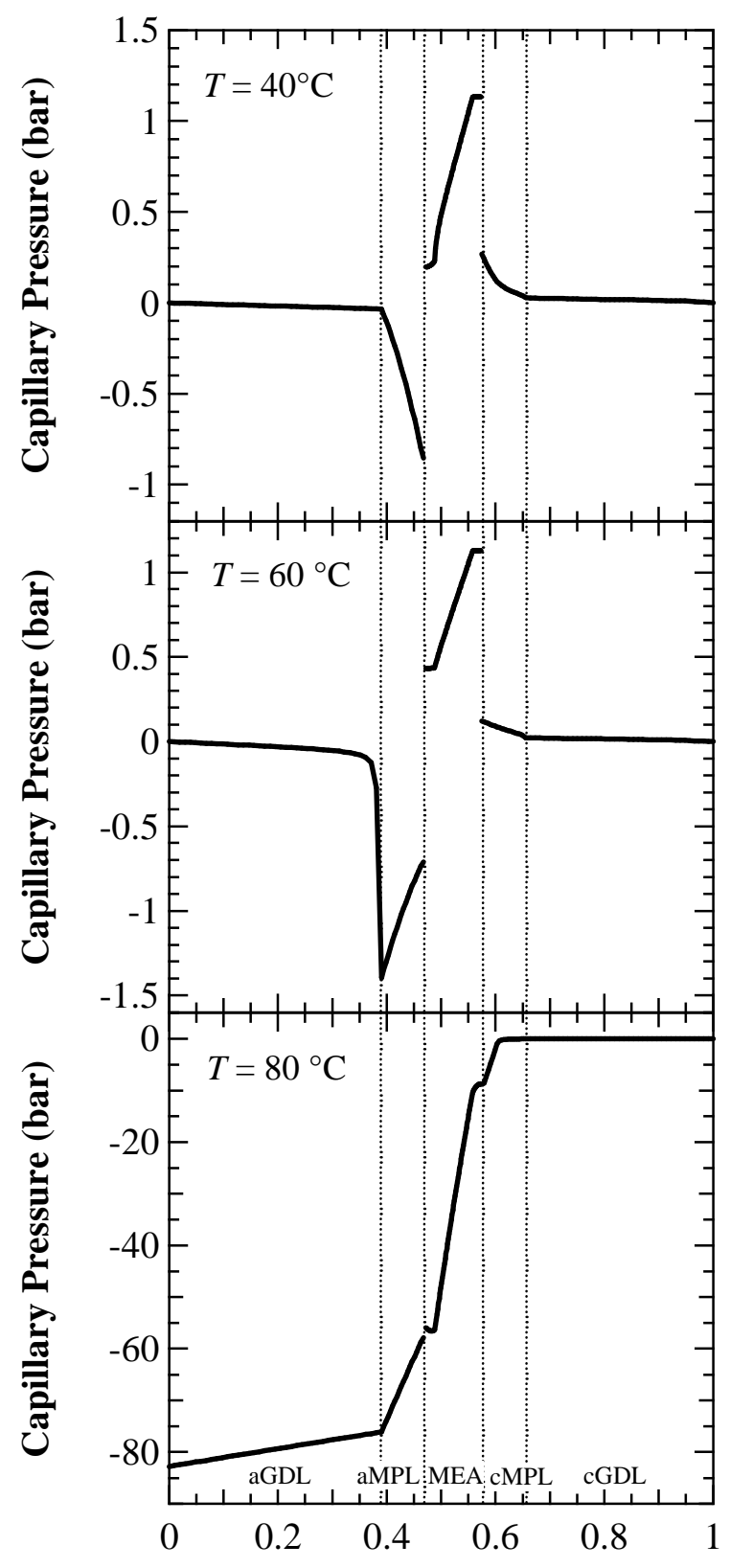

Dimensionless Position

Figure 4. Simulated capillary-pressure profiles from the anode to the cathode GDL / gaschannel interfaces for $0.75 \mathrm{~A} \mathrm{~cm}^{-2}$ at 40,60 , and $80^{\circ} \mathrm{C}$ with $100 \% \mathrm{RH}$ feed gases. The break is due to different meaning of capillary pressure in the membrane. The lower the capillary pressure, the lower the water content, with larger negative values (outside of the membrane) being associated with water at its residual saturation (i.e., movement is by water-vapor alone). 


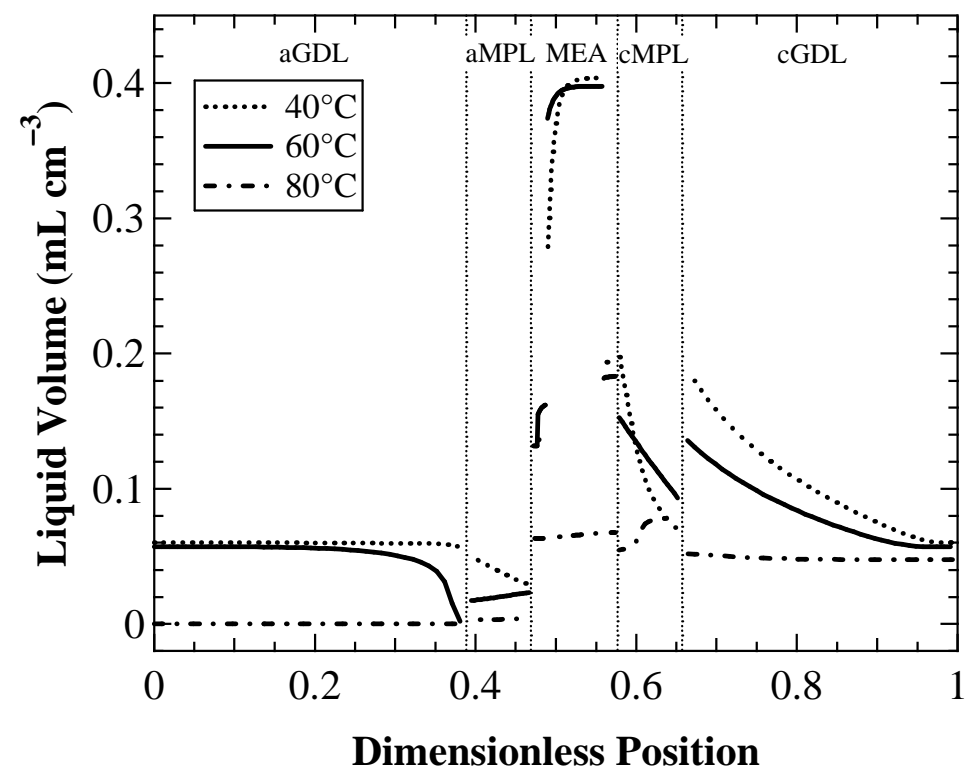

Figure 5. Liquid-volume profiles for the cases in Figure 4. For the conversion, symmetric layers are assumed (e.g., same GDL properties on the anode and cathode), with the same hydrophilic and hydrophobic pore-size distributions, $\varepsilon_{\mathrm{GDL}}=0.6, \varepsilon_{\mathrm{MPL}}=\varepsilon_{\mathrm{CL}}=0.3, \varepsilon_{\mathrm{Mem}}=0.4$ in the catalyst layers, $f_{\mathrm{HI}, \mathrm{GDL}}=0.1$, and $f_{\mathrm{HI}, \mathrm{CL}}=f_{\mathrm{HI}, \mathrm{MPL}}=0.06$. 


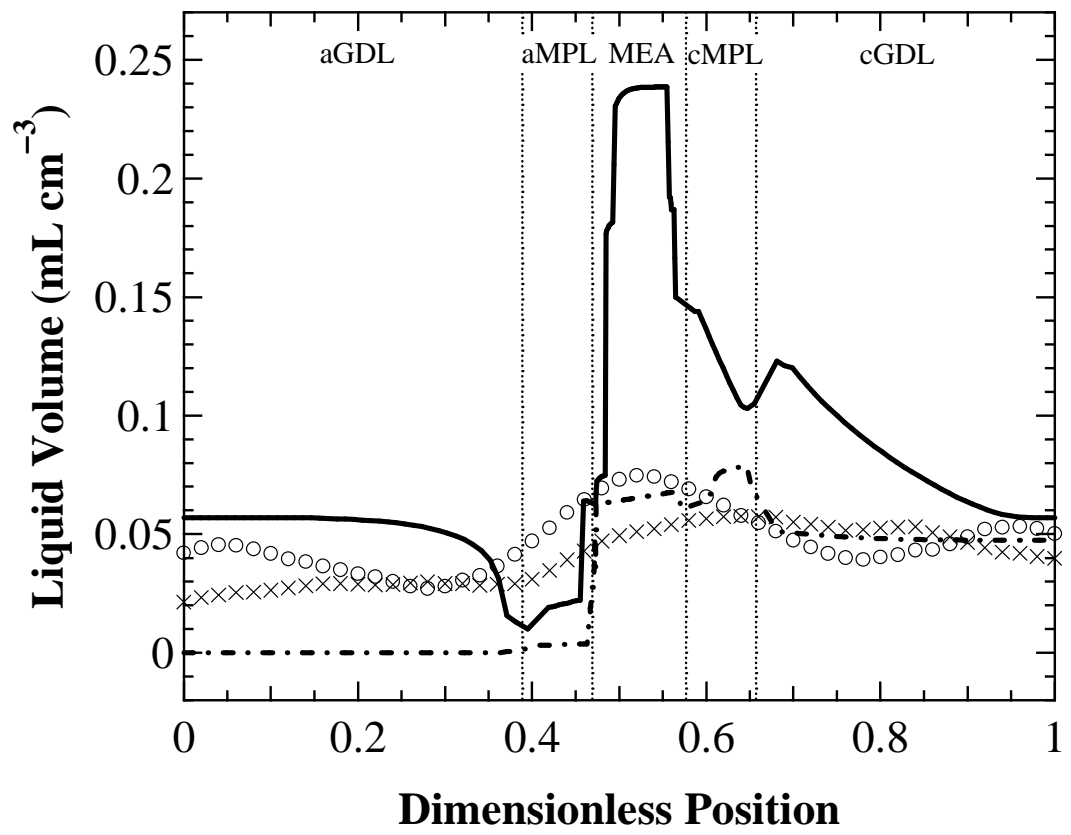

Figure 6. Simulated (see Figure 5) liquid-volume profiles for the 60 and $80^{\circ} \mathrm{C}$ cases assuming a phase-in-contact membrane model and smoothing of the interfaces. The curves are overlaid on the experimental data from Figure 2. 


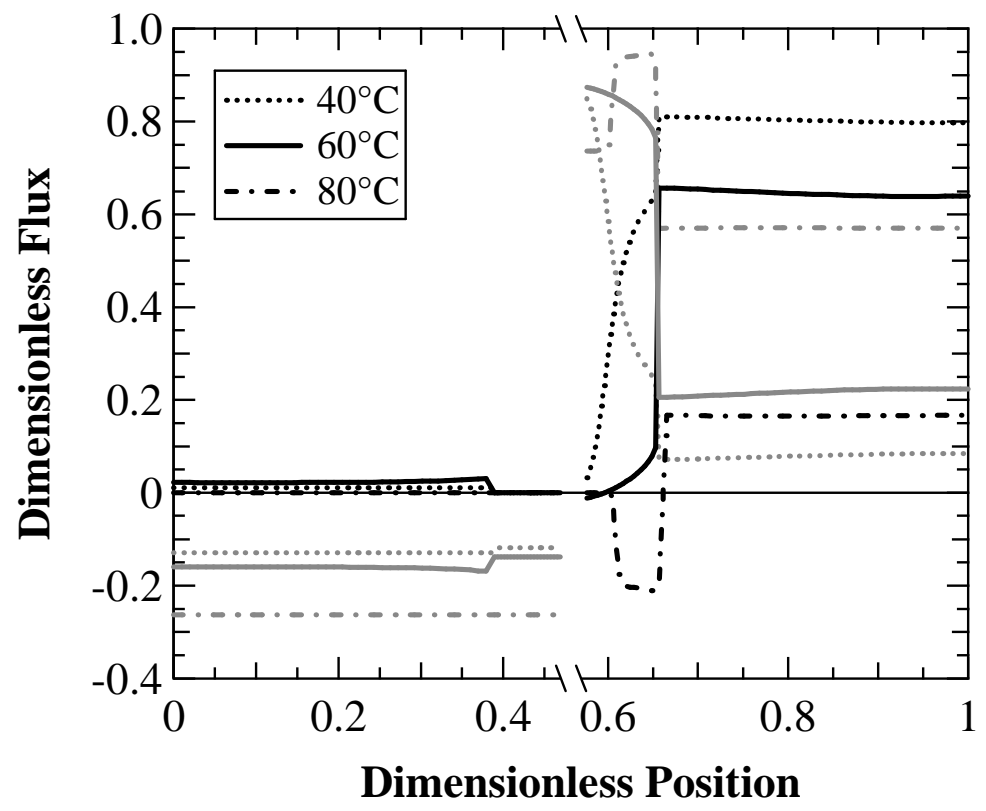

Figure 7. Liquid- (black) and vapor- (grey) water-flux profiles for the anode and cathode diffusion medium (GDLs and MPLs) as a function of cell temperature for 100\% RH feed gases. The fluxes are made dimensionless by dividing by the water production rate at $0.75 \mathrm{~A} \mathrm{~cm}^{-2}$. 


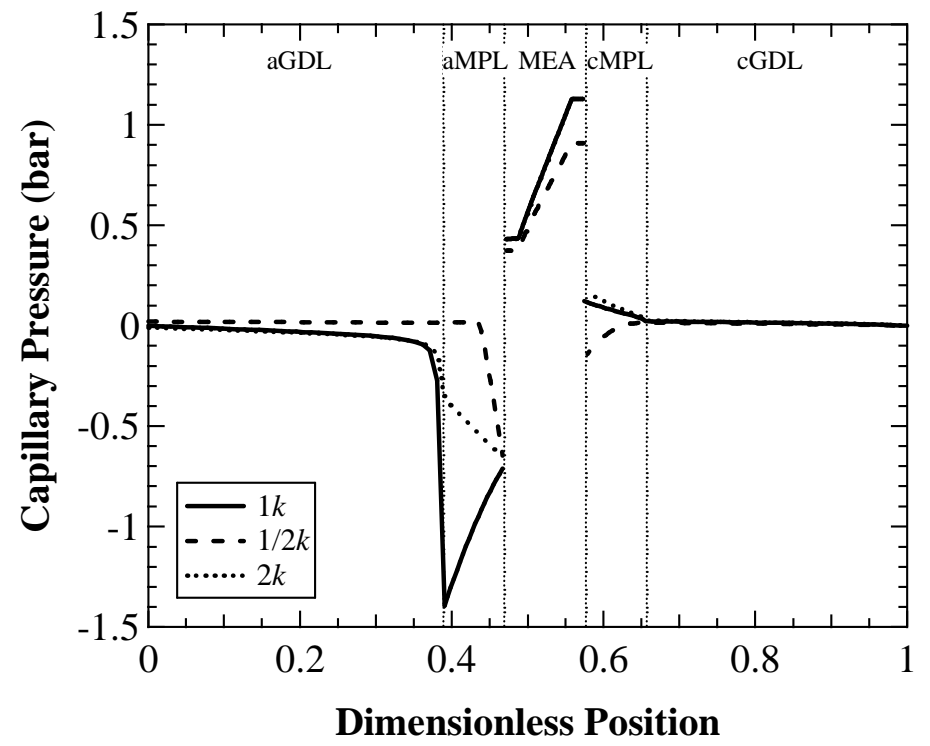

Figure 8. Simulated capillary-pressure profiles at $0.75 \mathrm{~A} \mathrm{~cm}^{-2}$ and $60^{\circ} \mathrm{C}$ for base, double, and half values of the GDL and MPL effective thermal conductivities with $100 \%$ RH feeds. 


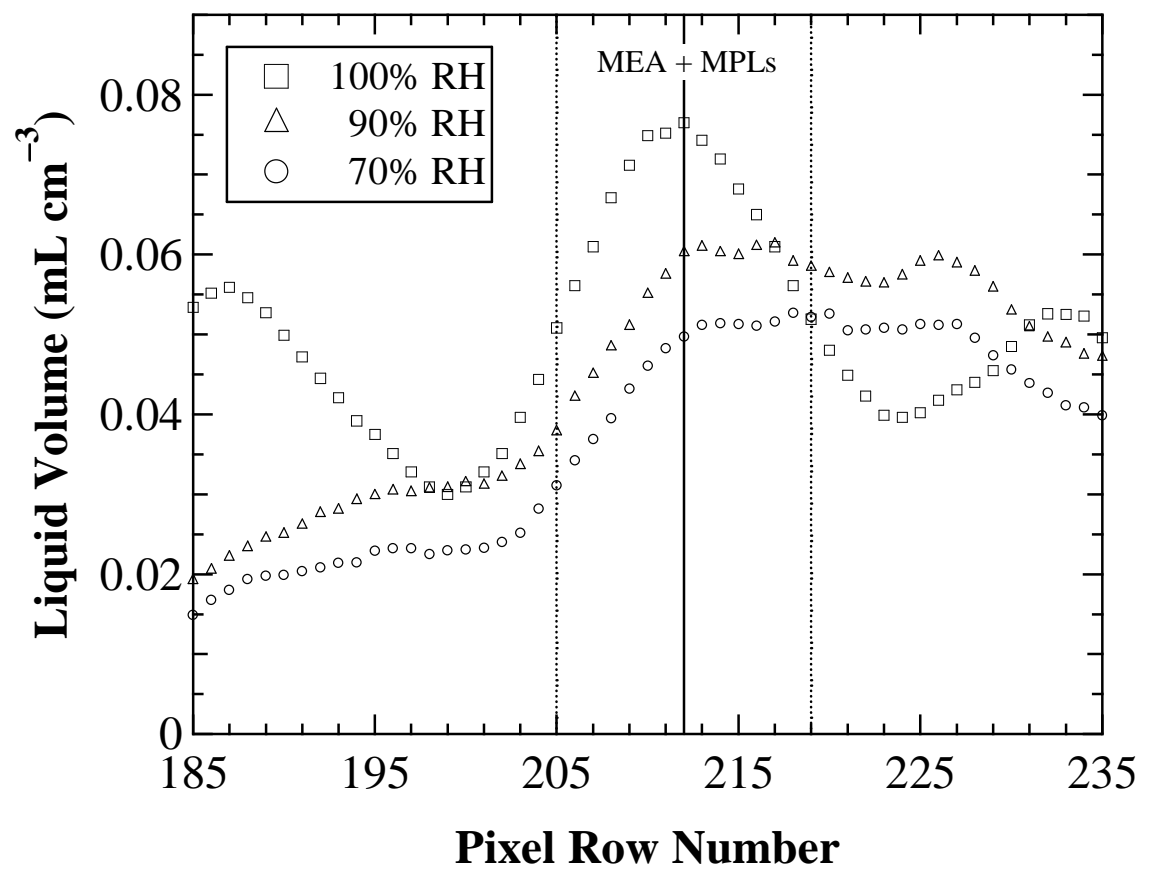

Figure 9. Through-plane water-volume fractions for gas feeds of 100, 90, and $70 \% \mathrm{RH}$ at $60{ }^{\circ} \mathrm{C}$ for the anode and cathode respectively at $1 \mathrm{~A} \mathrm{~cm}^{-2}$. 